\title{
Asteroid target selection for the new Rosetta mission baseline
}

\section{Lutetia and 2867 Steins *}

\author{
M. A. Barucci ${ }^{1}$, M. Fulchignoni ${ }^{1}$, S. Fornasier ${ }^{2}$, E. Dotto ${ }^{3}$, P. Vernazza ${ }^{1}$, M. Birlan ${ }^{4}$, R. P. Binzel ${ }^{5}$, J. Carvano ${ }^{1}$, \\ F. Merlin ${ }^{1}$, C. Barbieri ${ }^{2}$, and I. Belskaya ${ }^{6}$ \\ 1 LESIA, Observatoire de Paris, 92195 Meudon Principal Cedex, France \\ e-mail: antonella.barucci@obspm.fr \\ 2 Astronomy Department, Padova University, Vicolo dell'Osservatorio 2, 35122 Padova, Italy \\ 3 INAF - Osservatorio Astronomico di Roma, via Frascati 33, 00040 Monte Porzio Catone, Roma, Italy \\ ${ }^{4}$ IMCCE, Observatoire de Paris, 75014 Paris, France \\ 5 Dep. of Earth, Atmosph. and Planetary Sciences, Massachusetts Institute of Technology, Cambridge, MA 02139, USA \\ ${ }^{6}$ Astronomical Observatory of Kharkiv National University, Ukrania
}

Received 22 June 2004 / Accepted 18 September 2004

\begin{abstract}
The new Rosetta mission baseline to the comet 67P/Churyumov-Gerasimenko includes two asteroid fly-bys. To help in target selection we studied all the candidates of all the possible scenarios. Observations have been carried out at ESO-NTT (La Silla, Chile), TNG (Canaries), and NASA-IRTF (Hawaii) telescopes, in order to determine the taxonomy of all the candidates. The asteroid targets were chosen after the spacecraft interplanetary orbit insertion manoeuvre, when the available total amount of $\Delta V$ was known. On the basis of our analysis and the available of $\Delta V$, we recommended to the ESA Science Working Group the asteroids 21 Lutetia and 2867 Steins as targets for the Rosetta mission. The nature of Lutetia is still controversial. Lutetia's spectral properties may be consistent with a composition similar to carbonaceous chondrite meteorites. The spectral properties of Steins suggest a more extensive thermal history. Steins may have a composition similar to relatively rare enstatite chondrite/achondrite meteorites.
\end{abstract}

Key words. minor planets, asteroids - techniques: spectroscopic

\section{Introduction}

In 1993, the European Space Agency (ESA) selected the Rosetta mission including a rendezvous with in situ investigation of a comet and at least one (or more probably two) fly-bys of asteroids. The aim of the mission is to investigate the origin of the Solar System through the composition of planetesimals and their origin over the last 4.6 billion years.

In January 2003, ESA decided to postpone the launch of the spacecraft due to problems with the launcher (Barucci et al. 2004). The new baseline mission included a long orbital rendezvous with the 67P/Churyumov-Gerasimenko comet nucleus and one or two asteroid fly-bys. Several single or double flyby scenarios were designed (Table 1), depending on the total $\Delta V$ available after the spacecraft interplanetary orbit insertion manoeuvre. In December 2003, the asteroid 2513 Baetsle was included on the basis of the pre-launch resource budget as baseline target, due to the minimum extra $\Delta V$ required to reach it (only $19 \mathrm{~m} / \mathrm{s}$ ).

^ Based on observations collected at ESO-La Silla, NASA/IRTF and TNG-Canaries.
Table 1. Double and single asteroid mission opportunity with fly-by between 2008 and 2010. The listed $\Delta V$ values were estimated before the launch.

\begin{tabular}{lr|lr}
\hline \hline Double fly-by & $\Delta V \mathrm{~m} / \mathrm{s}$ & Single fly-by & $\Delta V \mathrm{~m} / \mathrm{s}$ \\
\hline Rhodia + Lutetia & 159 & Lutetia & 125 \\
Steins + Lutetia & 139 & Rhodia & 97 \\
Luichewoo + Lutetia & 129 & Steins & 57 \\
Baetsle + Izvekov & 73 & Sofala & 101 \\
Baetsle + Fogelin & 79 & Fogelin & 18 \\
Luichewoo + Izvekov & 32 & Baetsle & 19 \\
Rhodia + Izvekov & 112 & Luichewoo & 32 \\
Rhodia + Fogelin & 113 & Carrera & 102 \\
Steins + Izvekov & 77 & Izvekov & 15 \\
Sofala + Izvekov & 146 & & \\
Steins + Fogelin & 83 & & \\
Luichewoo + Fogelin & 35 & & \\
\hline
\end{tabular}

The mission was launched successfully on March 2nd 2004. The spacecraft started its journey to the comet $67 \mathrm{P} /$ Churyumov-Gerasimenko that will be reached on 2014, after three Earth and one Mars gravity assisted swing-bys and 
Table 2. Observational circumstances of asteroid targets for the Rosetta mission.

\begin{tabular}{|c|c|c|c|c|c|c|c|c|}
\hline Object & Night & $\begin{array}{l}\text { UT-start } \\
\text { (hh:mm) }\end{array}$ & $\begin{array}{l}T_{\exp } \\
(\mathrm{s})\end{array}$ & Tel. & Instr. & Grism/prism & $\begin{array}{c}\text { Slit } \\
(\operatorname{arcsec})\end{array}$ & airm. \\
\hline 437 Rhodia & 25 Jan. 04 & $15: 35$ & 600 & IRTF & SpeX & $0.8-2.5 \mu \mathrm{m}$ & 0.8 & 1.30 \\
\hline 437 Rhodia & 03 Mar. 04 & 10:07 & 960 & IRTF & SpeX & $0.8-2.5 \mu \mathrm{m}$ & 0.8 & 1.17 \\
\hline 1393 Sofala & 25 Jan. 04 & $15: 00$ & 720 & IRTF & SpeX & $0.8-2.5 \mu \mathrm{m}$ & 0.8 & 1.05 \\
\hline 2513 Baetsle & 25 Jan. 04 & $14: 21$ & 1320 & IRTF & SpeX & $0.8-2.5 \mu \mathrm{m}$ & 0.8 & 1.10 \\
\hline 2867 Steins & 25 Jan. 04 & $13: 21$ & 1100 & IRTF & SpeX & $0.8-2.5 \mu \mathrm{m}$ & 0.8 & 1.02 \\
\hline 3050 Carrera & 25 Jan. 04 & $11: 00$ & 1200 & IRTF & SpeX & $0.8-2.5 \mu \mathrm{m}$ & 0.8 & 1.08 \\
\hline 21 Lutetia & 26 May 04 & $10: 13$ & 660 & NTT & EMMI & GRISM $1(0.4-0.95 \mu \mathrm{m})$ & 1.5 & 1.55 \\
\hline 2513 Baetsle & 30 Jan. 04 & $8: 40$ & 960 & NTT & EMMI & GRISM $1(0.4-0.95 \mu \mathrm{m})$ & 1.5 & 1.13 \\
\hline 2867 Steins & 29 Jan. 04 & $7: 58$ & 720 & NTT & EMMI & GRISM $1(0.4-0.95 \mu \mathrm{m})$ & 1.0 & 1.40 \\
\hline 2867 Steins & 25 May 04 & $23: 28$ & 1200 & NTT & EMMI & GRISM $1(0.4-0.95 \mu \mathrm{m})$ & 1.5 & 1.41 \\
\hline 3050 Carrera & 30 Jan. 04 & $6: 50$ & 960 & NTT & EMMI & GRISM $1(0.4-0.95 \mu \mathrm{m})$ & 1.5 & 1.29 \\
\hline 5538 Luichewoo & 29 Jan. 04 & $7: 00$ & 1920 & NTT & EMMI & GRISM $1(0.4-0.95 \mu \mathrm{m})$ & 1.0 & 1.20 \\
\hline 437 Rhodia & 29 Feb. 04 & $02: 42$ & 180 & TNG & DOLORES & LR-R $(0.51-0.95 \mu \mathrm{m})$ & 1.5 & 1.28 \\
\hline 1393 Sofala & 29 Feb. 04 & 03:46 & 360 & TNG & DOLORES & LR-R $(0.51-0.95 \mu \mathrm{m})$ & 1.5 & 1.09 \\
\hline 2513 Baetsle & 29 Feb. 04 & 04:06 & 1200 & TNG & DOLORES & LR-R $(0.51-0.95 \mu \mathrm{m})$ & 1.5 & 1.31 \\
\hline 3418 Izvekov & 29 Feb. 04 & 02:18 & 480 & TNG & DOLORES & LR-R $(0.51-0.95 \mu \mathrm{m})$ & 1.5 & 1.07 \\
\hline 1393 Sofala & 1 Mar. 04 & 05:08 & 480 & TNG & NICS & AMICI $(0.8-2.5 \mu \mathrm{m})$ & 1.5 & 1.25 \\
\hline 2513 Baetsle & 2 Mar. 04 & $02: 44$ & 1920 & TNG & NICS & AMICI $(0.8-2.5 \mu \mathrm{m})$ & 1.5 & 1.19 \\
\hline 3418 Izvekov & 2 Mar. 04 & 01:38 & 1920 & TNG & NICS & AMICI $(0.8-2.5 \mu \mathrm{m})$ & 1.5 & 1.08 \\
\hline 5538 Luichewoo & 2 Mar. 04 & 03:51 & 1920 & TNG & NICS & AMICI $(0.8-2.5 \mu \mathrm{m})$ & 1.5 & 1.32 \\
\hline
\end{tabular}

the asteroid fly-bys. Due to the optimal launch conditions, the available remaining $\Delta V$ is enough to fly-by two asteroids. To help in the selection of the best targets for scientific return, we observed all the possible candidates by visible (V) and nearinfrared (NIR) spectroscopy. In this paper we report the results of the observational campaign as well as the characterization of the selected targets.

\section{Observations and data reduction}

Visible and near-infrared spectroscopic observations were performed at the ESO $3.5 \mathrm{~m}$ New Technology Telescope (NTT, La Silla, Chile), the $3.5 \mathrm{~m}$ Telescopio Nazionale Galileo (TNG, La Palma, Spain), and the $3 \mathrm{~m}$ NASA Infrared Telescope Facility (IRTF, Hawaii, USA). The circumstances of these observations are summarized in Table 2. During each observing run we also acquired bias, flat-field, calibration lamp and several solar analog star spectra at different intervals throughout the night.

The observations at IRTF were performed in remote mode from the Observatoire de Paris-Meudon (Binzel et al. 2004; Birlan et al. 2004). The NIR acquisition procedure was the same both for the TNG and IRTF telescopes, consisting of cycle series of 4 images each (ABBA cycle). The images had an exposure time of $120 \mathrm{~s}$ each, and were taken in two different positions along the slit, named A and B, offsetting the telescope. This technique obtains near-simultaneous images for sky and bias subtraction. The ABBA cycles were repeated several times for the fainter objects. The total exposure time for each object is indicated in Table 2.

Spectra were reduced using standard data reduction procedures with the software package Midas (e.g. Fornasier \& Lazzarin 2001; Fornasier et al. 2003). The asteroid relative reflectance has been obtained by division of the asteroid spectrum with that one of the solar analog star closest in time and airmass to the asteroid. For the IRTF data, the asteroid spectrum has been divided by a "superstar", created by summing appropriately weighted contributions of several observed analog stars, corrected for atmospheric extinction and computed at the same airmass of the asteroids.

\section{Data analysis and discussion}

We obtained the first V+NIR spectra of seven out of the nine asteroid candidates listed in Table 1 (437 Rhodia, 1393 Sofala, 2513 Baetsle, 2867 Steins, 3050 Carrera, 3418 Izvekov, and 5538 Luichewoo). A visible spectrum of 21 Lutetia has been also obtained and reported in Fig. 2. The NIR spectra of Lutetia and Fogelin have been recently published by our group (Birlan et al. 2004).

All the objects reported in Fig. 1 seem to belong to the $\mathrm{S}$ taxonomic class because of well defined 1 and $2 \mu \mathrm{m}$ silicate bands. Spectra shown in Fig. 2 have flatter behaviors. The presence of the absorption band at $0.9 \mu \mathrm{m}$ in the spectra of Rhodia together with its high albedo value (0.56, IRAS data: Tedesco et al. 1992) suggests a possible classification as an E-type asteroids; Carrera has a similar spectral behaviour with the presence of the peculiar $0.9 \mu \mathrm{m}$ band and might also be an $\mathrm{E}$ type asteroid, even if the knowledge of its albedo is necessary to make a definitive taxonomic class assignment. Izvekov has a very peculiar flat spectrum, typical of primitive dark objects like B- or C-type. The spectral behaviour of Steins seems to suggest that it belongs to E-type class. Spectra of the same object obtained during different epochs are essentially the same. For Sofala a visible spectrum was 


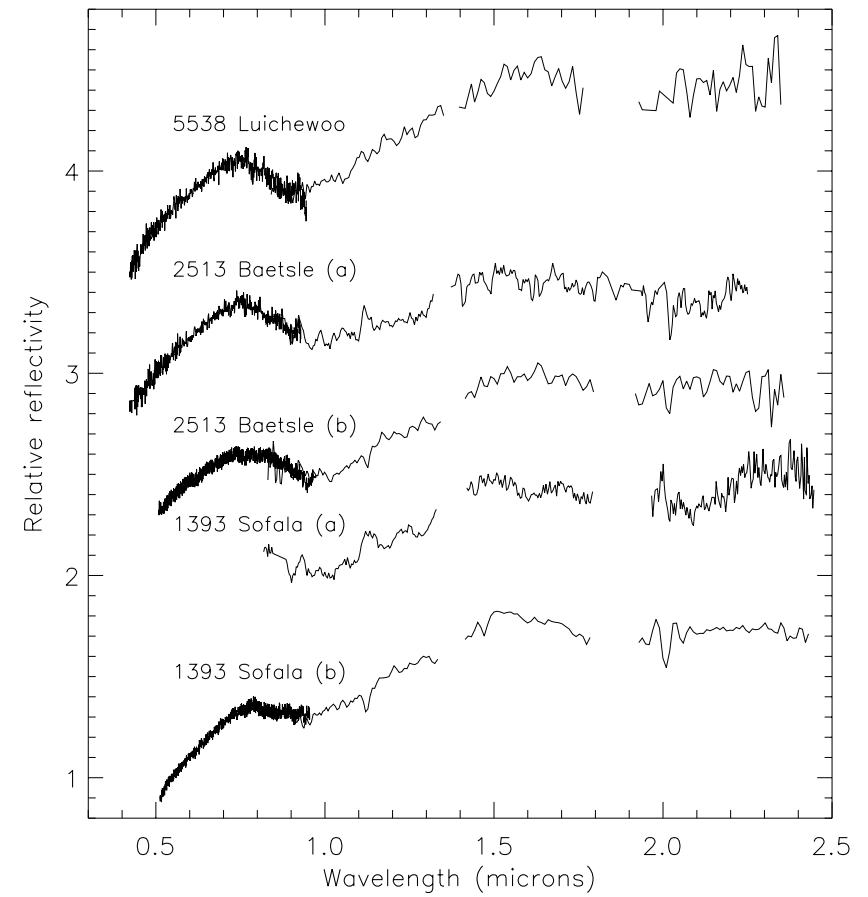

Fig. 1. V and NIR spectra of Luichewoo, Baetsle, and Sofala. For Baetsle and Sofala, the spectra (a) include V data obtained at NTT and NIR spectra obtained at IRTF, while spectra (b) have been obtained at TNG. All the spectra are normalized at $0.55 \mu \mathrm{m}$ and shifted by 0.7 for clarity. All these objects have been classified as S-type.

already published by Xu et al. (1995). We confirm their S-type classification.

\section{Target selection and characterization}

Analyzing the list of possible targets and the relative available data, we strongly recommended the inclusion of asteroid 21 Lutetia in the Rosetta baseline because of its high interest. In fact it is the only object of the list which will allow the radio science experiment to measure the mass, and consequently to deduce the bulk density.

After the insertion manoeuvre of Rosetta into its interplanetary orbit, the available $\Delta V$ for the asteroid encounters was enough to allow the selection of any pair of asteroids listed in Table 1 for a double fly-by, except for the RhodiaLutetia one. The two remaining pairs which include 21 Lutetia are Steins-Lutetia and Luichewoo-Lutetia. As Luichewoo is a S-type ( $\mathrm{S}$ class asteroids have already been visited by space missions), we exclude it from the possible baseline.

On the basis of the obtained spectra, we suggested Lutetia and Steins as the best asteroid targets for the Rosetta mission. In fact, these asteroids seem to be peculiar and show spectral behaviors different from the previous asteroid targets of space missions.

21 Lutetia was discovered in 1852 by $\mathrm{H}$. Goldchmidt at the Paris Observatory. The name Lutetia derives from the Roman name of Paris (Lutetia Parisorum) and was given to the asteroid to honour the French capital. 21 Lutetia is the largest asteroid out of the possible candidates (IRAS diameter of $95.8 \pm 4.1 \mathrm{~km})$. The main orbital and physical characteristics

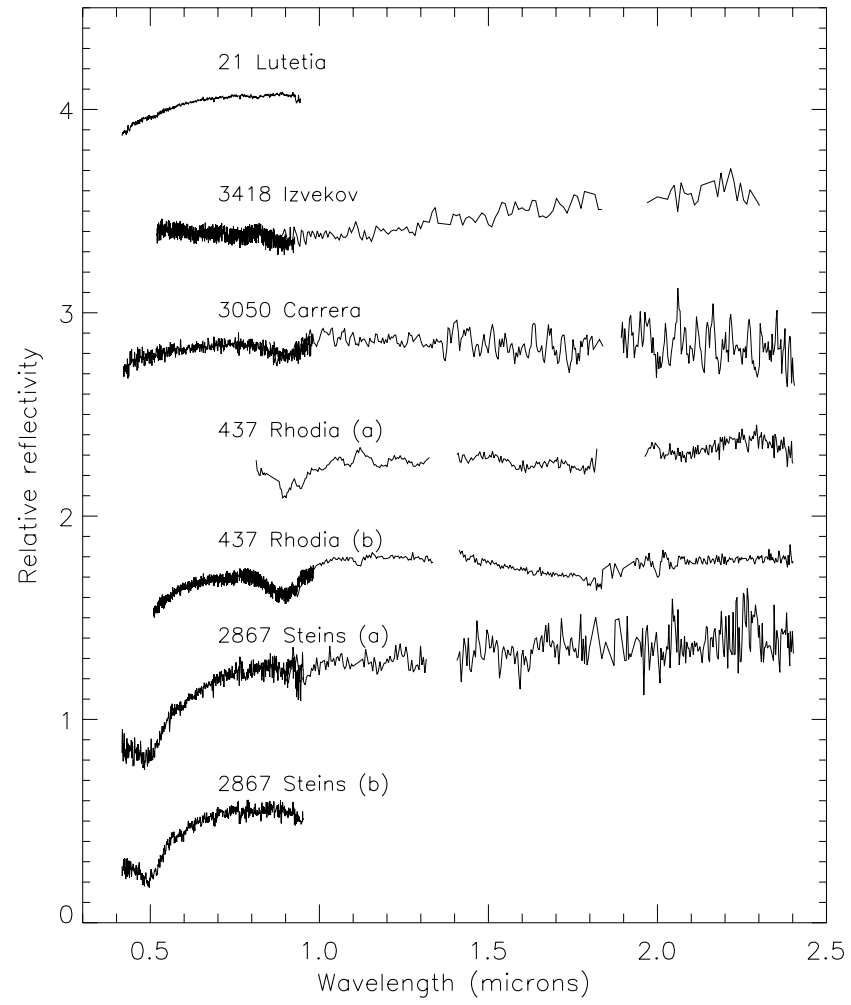

Fig. 2. V spectrum of Lutetia, and V and NIR spectra of Izvekov, Carrera, Rhodia, and Steins. The NIR spectrum (a) of Rhodia has been obtained on January 2004, while the NIR spectrum (b) has been obtained on March 2004. The V spectrum (b) of Steins has been obtained on May 2004. All the spectra are normalized at $0.55 \mu \mathrm{m}$ and shifted by 0.6 for clarity.

are listed in Table 3. The rotational period of $8.17 \pm 0.01 \mathrm{~h}$ has been determined by Zappalá et al. (1984). The spin vector direction of Lutetia has been computed by several authors (see Magri et al. 1999 for details) giving different pole solutions. The prograde rotation state was in agreement with the different methods and determinations. Torppa et al. (2003), analysing all the available lightcurves, computed pole coordinates and a model with sharp and irregular shape (see Table 3).

Because of the high IRAS albedo, Lutetia was previously classified as M-type by Barucci et al. (1987) and Tholen (1989) and was supposed to be a parent body of iron meteorites. Hiroi et al. (1993) found a good fit with the M meteorite Mundrabilla under particular ad hoc conditions. Bus \& Binzel (2002) proposed for it a new class $\mathrm{X}_{k}$. Further observations have shown that its infrared spectrum is unusually flat compared to other M asteroids (Howell et al. 1994; Burbine \& Binzel 2000). Moreover the observations obtained at IRTF (Birlan et al. 2004) suggested a similarity with the carbonaceous chondrite spectra which characterize the C-type asteroid. Busarev et al. (2004) and Lazzarin et al. (2004), obtained several V spectra showing rotational phase variation with possible presence of features at 0.44 and $0.67 \mu \mathrm{m}$ probably associated to hydrated silicates.

Many other characteristics seem to be in agreement with the carbonaceous chondrite analogy: the polarimetric properties (Belskaya \& Lagerkvist 1996), lower radar albedo (Magri et al. 1999), and the $3 \mu \mathrm{m}$ absorption feature diagnostic of water 
Table 3. Orbital and physical characteristics of 21 Lutetia.

\begin{tabular}{ll}
\hline \hline Semimajor axis (AU) & 2.435 \\
Eccentricity & 0.164 \\
Inclination (deg) & 3.064 \\
Taxonomic type & $\mathrm{C}($ or M) \\
Synodical rotation period (h) & $8.17 \pm 0.01$ \\
Absolute magnitude & 7.294 \\
Slope parameter & 0.110 \\
IRAS D (km) & $95.5 \pm 4.1$ \\
IRAS albedo & $0.221 \pm 0.20$ \\
\hline Pole solution from radar observations (Magri et al. 1999): & \\
$\lambda_{1}=228^{\circ} \pm 11^{\circ} \beta_{1}=+13^{\circ} \pm 5^{\circ}$ & $\lambda_{2}=48^{\circ} \pm 11^{\circ} \beta_{2}=+5^{\circ} \pm 5^{\circ}$ \\
Sense of rotation & Prograde \\
$2 a \times 2 b \times 2 c$ & $130 \times 104 \times 74 \mathrm{~km}$ \\
\hline Pole solution from lightcurve analysis (Torppa et al. 2003): & \\
$\lambda_{1}=220^{\circ} \pm 10^{\circ} \beta_{1}=+3^{\circ} \pm 10^{\circ}$ & $\lambda_{2}=39^{\circ} \pm 10^{\circ} \beta_{2}=+3^{\circ} \pm 10^{\circ}$ \\
$a / b=1.2 ; b / c=1.2$ & \\
\hline
\end{tabular}

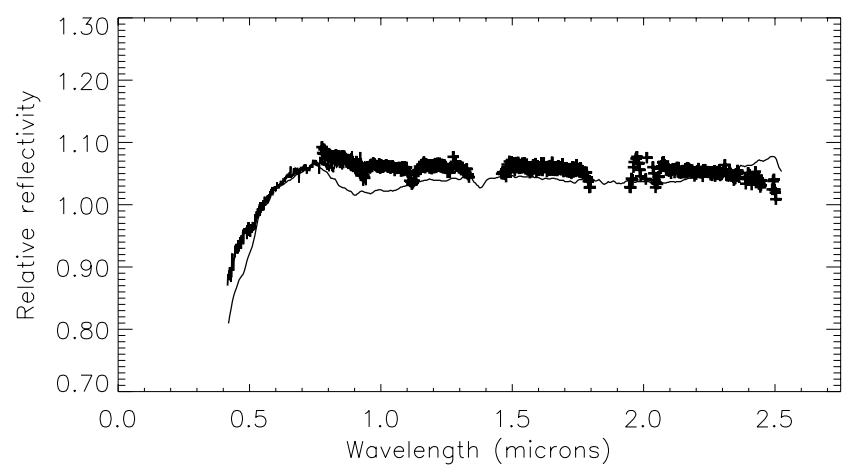

Fig. 3. Comparison of the Lutetia spectra (our V spectrum + the NIR one by Birlan et al. 2004) and the spectra of the CV3 carbonaceous chondrite meteorite Vigarano.

of hydration (Rivkin et al. 2000). All these evidences suggest that Lutetia is an atypical M-type object. In Fig. 3 the NIR spectra of Lutetia by Birlan et al. (2004) with our visible spectrum is reported and compared with the spectrum of the CV3 carbonaceous chondrite Vigarano.

All of the information available for Lutetia are consistent with a primitive composition, except the high IRAS albedo value. New albedo determinations are needed to eliminate the doubts about the taxonomic classification of Lutetia, or to further enhance our view of this unusual object.

2867 Steins, discovered on 1969 by N. Chernykh at Nauchnyj, is a very small object and only few of its properties are known (Table 4). Assuming an albedo in the range $0.04-0.40$, a diameter of $17.5-5.5 \mathrm{~km}$ can be estimated. Its rotational period of $6.06 \pm 0.05 \mathrm{~h}$ and a lightcurve amplitude of 0.2 mag has been recently determined by Hicks \& Bauer (2004).

The obtained V and NIR spectra (Figs. 2 and 4) show a strong feature at about $0.5 \mu \mathrm{m}$, a weaker feature at about $0.96 \mu \mathrm{m}$ and a flat and featureless behaviour over $1 \mu \mathrm{m}$. This spectral behaviour is very similar to E-type asteroids, even if it is necessary that the albedo, still to be determined, would be larger than 0.3 to classify Steins as an E-type.

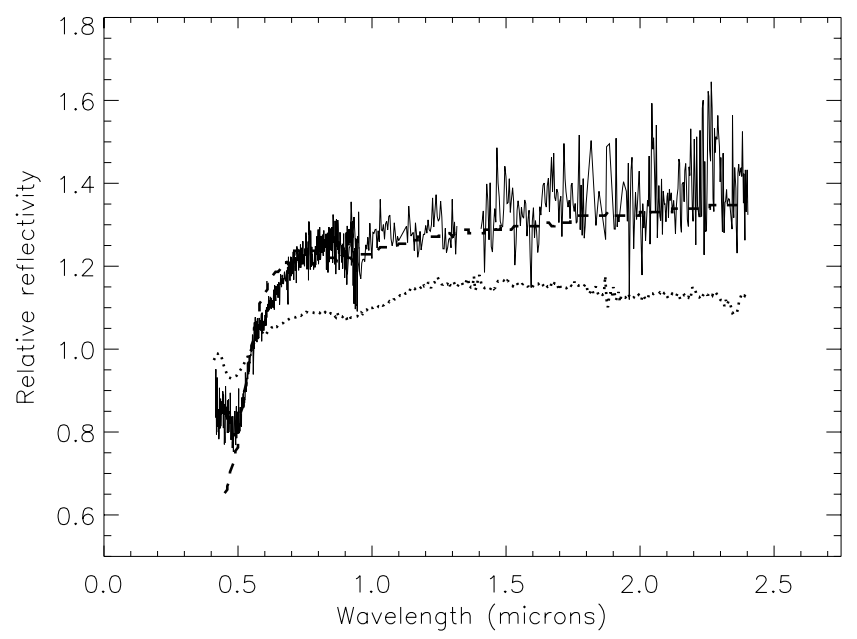

Fig. 4. Comparison of the spectrum of Steins and the spectra of the EL6 enstatite chondrite Atlanta (dashed line), and the enstatite achondrite (aubrite) ALH 78113 (dotted line). All the spectra are normalized at $0.55 \mu \mathrm{m}$.

Table 4. Orbital and physical characteristics of 2867 Steins.

\begin{tabular}{ll}
\hline \hline Semimajor axis (AU) & 2.364 \\
Eccentricity & 0.146 \\
Inclination (deg) & 9.944 \\
Taxonomic type & $\mathrm{E}$ \\
Synodical rotation period (h) & $6.06 \pm 0.05$ \\
Absolute magnitude (with & 12.561 \\
slope par. assumed $=0.15)$ & \\
\hline
\end{tabular}

E-type objects are thought to be differentiated bodies which experienced significant heating.

Analyzing the $0.5 \mu \mathrm{m}$ feature observed in the spectra of some E-type asteroids, Burbine (2000) suggested that some kind of sulfides, such as troilite or pyrrhotite, could produce this feature. Troilite is a rare sulfide $(\mathrm{FeS})$, abundant in meteorites. It is a known constituent of the aubrites but it is also a very dark agent not easily compatible with the high albedo of E-type asteroids (Fornasier \& Lazzarin 2001). Moreover 
the spectra available in literature of meteoritic and synthetic samples of troilite show quite different spectral behaviours. Also the band at about $0.5 \mu \mathrm{m}$ changes in wavelength position and shape, probably due to the different crystal structure of each samples.

The spectral behaviour of Steins as shown in Figs. 2 and 4 is similar to that of the E-type asteroid 64 Angelina. According to the classification of Gaffey \& Kelley (2004) and Clark et al. (2004), Steins would belong to the same group of Angelina, and its surface composition would include silicates and sulfides, like oldhamite (CaS). The presence of oldhamite on the surface of Angelina, has been also suggested by Burbine et al. (2002). Gaffey \& Kelley (2004) suggested that this group of objects (also called E[II] subtype) may have surfaces composed of partial melts derived from E-chondrite-like parent bodies.

In order to investigate the surface composition of Steins we compared its V and NIR spectrum with the spectra of many meteorites available in literature. In Fig. 4 the spectrum of Steins is overlain onto with the spectra of an enstatite achondrite, and an enstatite chondrite. The enstatite chondrite Atlanta (EL6) presents a spectrum that fits very well the observed spectral behaviour of Steins with the exception of the feature at about $0.5 \mu \mathrm{m}$. The enstatite achondrite ALH 78113 (aubrite) presents spectral features at $0.5 \mu \mathrm{m}$ and 0.96 similar to those of Steins' spectrum but with a flatter behaviour at $\lambda>0.6 \mu \mathrm{m}$.

The differences between asteroid spectra and those of meteorites and mineral assemblages could be due to the effects of space weathering (caused to long cosmic ray exposure) and of surface collisional processing (porosity, grain size distribution, aging of materials...).

\section{Conclusions}

On the basis of i) the results obtained by the spectroscopic observations; and ii) on the analysis of all the known physical properties of the candidates, we recommend target selection of 21 Lutetia and 2867 Steins. The Rosetta Science Working group supported our recommendation and the ESA's Rosetta Project included these asteroids in the new mission baseline. The fly-by with Steins will take place on September 5 th, 2008, with a relative velocity of $8.6 \mathrm{~km} \mathrm{~s}^{-1}$ and the one with 21 Lutetia on July 10th 2010 with a relative velocity of $15 \mathrm{~km} \mathrm{~s}^{-1}$. In particular, for the two selected candidates:

1. Lutetia represents the most interesting candidate, as it is the only one (among the possible targets) which will allow us to obtain, by radio science experiments, mass determination and density estimation. Moreover, if the primitive chondritic character of Lutetia will be confirmed, it will be well within the early Solar system scientific objectives of the mission.

2. After the choice of the first target, following the criteria to be within the available $\Delta V$ and to visit an interesting object, leads 2867 Steins as the best candidate for the other fly-by. Steins has relatively unusual spectral properties and may be classified as E-type object. Rosetta will perform the first in-situ exploration of a member of this rare class of objects, giving an answer to the question if its parent body underwent a total differentiation or a partial melting.

Ground-based observations are still needed to better define the physical and mineralogical characteristics of Lutetia and Steins. Further observations, in particular in the thermal infrared, are fundamental to know the albedo of both the objects. More information will allow us to optimize the science operations during the fly-bys.

\section{References}

Barucci, M. A., Capria, M. T., Coradini, A., \& Fulchignoni, M. 1987, Icarus, 72, 304

Barucci, M. A., Fulchignoni, M., Belskaya, I., et al. 2004, in The new Rosetta targets, ASSL (Kluwer), 69

Belskaya, I. N., \& Lagerkvist, C.-I. 1996, PSS, 44, 783

Binzel, R. P., Birlan, M., Bus, S. J., et al. 2004, PSS, 52, 291

Birlan, M., Barucci, M. A., Vernazza, P., et al. 2004, New Astron., 9, 343

Burbine, T. H. 2000, Ph.D. Thesis, MIT

Burbine, T. H., \& Binzel, R. P. 2002, Icarus, 159, 468

Burbine, T. H., McCoy, T. J., Nittler, L. R., et al. 2002, Meteo. Planet. Sci., 37, 1233

Bus, B., \& Binzel, R. 2002, Icarus 158, 146

Busarev, V., Bochokov, V., Prokof'eva, V., et al. 2004, in The new Rosetta targets, ASSL (Kluwer), in press

Clark, B. E., Bus, S. J., Rivkin, A. S., et al. 2004, J. Geo. Res., 109, 2001

Fornasier, S., \& Lazzarin, M. 2001, Icarus, 152, 127

Fornasier, S., Barucci, M. A., Binzel, R. P., et al. 2003, A\&A, 398, 327

Gaffey, M. J., \& Kelley, M. S. 2004, LPI XXXV, 1812

Hicks, M. D., \& Bauer, J. M. 2004, IAUC, 8315

Hiroi, T., Bell, J. F., Takeda, H., et al. 1993, Icarus, 102, 107

Howell, E. S., Merenyi, E., \& Lebofsky, L. A. 1994, J. Geo. Res., 99, 10848

Lazzarin, M., Marchi, S., Magrin, S., \& Barbieri, C. 2004, A\&A, 425, L25

Magri, C., Ostro, S. J., Rosema, D. K., et al. 1999, Icarus, 140, 379

Rivkin, A. S., Howell, E. S., Lebofsky, L. A., et al. 2000, Icarus, 145, 351

Tedesco, E. F., \& Veeder, G. F. 1992, in The IRAS Minor planet Survey, Tech. Rep. PL-TR-92-2049, Phillips Laboratory, Hanscom AF Base, MA

Tholen, D. 1989, in Asteroids II (Univ. of Arizona Press), 1139

Torppa, J., Kaasalainen, M., Michalowski, T., et al. 2003, Icarus, 164, 346

Xu, S., Binzel, R. P., Burbine, T. H., et al. 1995, Icarus, 115, 1

Zappalà, V., Di Martino, M., Knezevic, Z., et al. 1984, A\&A, 130, 208 